\title{
El río Meta y los proyectos para la integración de los Llanos Orientales colombianos, desde la Colonia hasta el siglo XXI ${ }^{1}$
}

\section{Resumen}

El Meta es un río lleno de sueños y esperanzas, que se han construido desde los primeros conquistadores y misioneros que recorrieron sus aguas hasta los grandes proyectos de infraestructura del siglo XXI. Desde La Colonia, la región de los Llanos que recorre el río Meta se configuró como frontera a causa del clima caliente, de los pobladores salvajes, y de los Andes como una barrera geográfica que los alejaba del centro administrativo; sin embargo, el río siempre fue pensado e imaginado como un eje integrador para incluir un extenso territorio a la civilización, al progreso y al desarrollo. Este artículo tiene como objetivo analizar diferentes visiones y proyectos para la integración de los Llanos Orientales colombianos, los cuales tienen como eje al río Meta, y cuyo objetivo fue cambiar la situación de frontera de la región. Aunque ninguno de los proyectos logró plenamente su objetivo, su ejecución permite evidenciar que la región ha sido pensada e intervenida siempre desde el exterior, sin considerar sus particularidades ni la de sus pobladores; lo que ha generado profundos cambios en el paisaje.

Palabras clave: Colombia, Orinoquia, Llanos Orientales, frontera.

Referencia para citar este artículo: ARDILA LUNA, Diana Carolina (2016). "El río Meta y los proyectos para la integración de los Llanos Orientales colombianos, desde la Colonia hasta el siglo XXI". En Anuario de Historia Regional y de las Fronteras. 21 (2). pp. 265-283.

Fecha de recepción: 24/11/2015

Fecha de aceptación: 29/02/2016

Diana Carolina Ardila Luna: Candidata a doctora en Antropología, Universidad de los Andes (Colombia). Magíster en Medio Ambiente y Desarrollo, Universidad Nacional de Colombia. Antropóloga, Universidad Nacional de Colombia. Correo electrónico: dc.ardila10@uniandes.edu.co.

\footnotetext{
${ }^{1}$ Este artículo hace parte del proyecto de tesis de doctorado en Antropología de la Universidad de los Andes titulado: El río Meta en el proyecto de navegabilidad e integración a la economía global: dinámicas y efectos, inscrito en el grupo Naturaleza y Sociedad de la Universidad de Los Andes.
} 


\title{
The Meta River and Projects for the Integration of the Colombian Eastern Plains, from Colonial Times to the $21^{\text {st }}$ Century
}

\begin{abstract}
The Meta River is full of hopes and dreams that have been built from the early conquerors and missionaries who plied its waters to the large infrastructure projects of the $21^{\text {st }}$ century. From the Colonial times, the Plains region, through which the Meta River runs, was established as a boundary because of hot weather, the "wild" dwellers, as well as the Andes range as a geographical barrier that pulled away from the administrative center; however, the river was always thought and imagined as an integrating axis to include a vast territory into civilization, progress and development. This article aims to analyze different views and projects for the integration of Colombia's Eastern Plains, whose axis is the Meta River, and whose objective was to change the border situation of the region. Although none of the projects fully achieved its goal, their execution makes evident that the region has always been planned and operated from the outside; and without considering its peculiarities or those of its inhabitants; and that situation has generated profound changes in the landscape.
\end{abstract}

Keywords: Colombia, Orinoquia, Llanos Orientales, Border.

\section{Resumo}

\section{O rio Meta e os projetos para a integração das Planícies Orientais colombianas, desde a Colônia até o século XXI}

O Meta é um rio cheio de sonhos e esperanças que foram construidas desde os primeiros conquistadores e missioneiros que recorreram suas águas até os grandes projetos de infraestrutura do século XXI. Desde a Colônia, a região de Los Llanos que recorre o rio Meta, foi configurada como fronteira por causa do clima quente, da população "selvagem" e dos Andes como uma barreira geográfica que os afastava do centro administrativo; sem embargo, o rio sempre foi pensado e imaginado como um eixo integrador para incluir um extenso território para a civilização, o progresso e o desenvolvimento. Este artigo tem como objetivo analisar diferentes visões e projetos para a integração das planícies orientais colombianas (Llanos Orientais), as quais têm como eixo o rio Meta e cujo objetivo foi transformar a situação de fronteira da região. Ainda que nenhum dos projetos conseguiu plenamente seu objetivo, sua execução permite evidenciar que a região foi pensada e intervinda sempre desde o exterior e sem considerar suas particularidades nem a dos seus habitantes, e tem gerado profundas transformações na paisagem.

Palavras chave: Colombia, Orinoquia, Llanos Orientales, fronteira. 


\section{Introducción: El río Meta en la estrategia de integración}

Caminos que andan es la descripción de Hipólito Pérez sobre el río Meta y tributarios². Pérez impresionado por la majestuosidad del Meta y su facilidad de navegación repite una idea que viajeros, exploradores, misioneros y gobernantes, anteriores y posteriores a él, han mencionado constantemente: el Meta es un camino para la integración de la región. El Meta es un río lleno de sueños y esperanzas, que se han construido desde los primeros conquistadores y misioneros que recorrieron sus aguas hasta los grandes proyectos de infraestructura del siglo XXI. Aunque desde temprano en la Colonia, la región de los Llanos que recorre el Meta se configuró como una zona de frontera a causa del clima caliente, de los pobladores salvajes, y de los Andes como una barrera geográfica que lo alejaba del centro administrativo ${ }^{3}$, el río ha sido pensado e imaginado como un eje integrador, un espacio privilegiado para incluir un extenso territorio a la civilización, al progreso y al desarrollo.

Este artículo tiene como objetivo analizar las diferentes visiones y proyectos para la integración de los Llanos Orientales colombianos, los cuales tienen como eje al río Meta, y cuyo objetivo era cambiar la situación de frontera de la región. Aunque ninguno de los proyectos logró plenamente su objetivo, su ejecución permite evidenciar que la región ha sido pensada e intervenida siempre desde el exterior, sin considerar sus particularidades ni la de sus pobladores, lo que ha generado profundos cambios en el paisaje $\mathrm{e}^{4}$. No es la intención de este artículo discutir la noción de frontera, pero sí es importante aclarar que este concepto sigue la propuesta de Rausch de frontera permanente $^{5}$ donde los Llanos se caracterizan por ser un espacio extenso, de clima caliente y vacío - poblado por salvajes-, punto que las visiones y proyectos analizados dan por sentado.

Las visiones son lecturas de un lugar en las que más allá de la descripción, el observador crea una imagen producto de la experiencia del paisaje observado, experiencia emocional, estética e imaginativa ${ }^{6}$. Imágenes, algunas, que logran permanecer en el tiempo y llegan a definir un lugar. Las visiones sobre el río llevaron a la formulación de proyectos específicos para la integración de la región en momentos determinado, entendiendo por proyecto una serie de ideas y prácticas preconcebidas que tienen

\footnotetext{
2 Pérez, Hipólito. Los jesuitas en Casanare (Bogotá: Prensas del Ministerio de Educación Nacional, 1952).

${ }^{3}$ Rausch, Jane. A tropical plains frontier: the Llanos of Colombia, 1531-1831 (Albuquerque: University of New Mexico Press, 1984).

${ }^{4}$ Para este trabajo se analizaron fuentes primarias impresas y secundarias para el análisis de la época colonial y el siglo XIX; para el siglo XX y XXI se analizaron fuentes primarias provenientes de diferentes archivos y algunas publicadas: Ministerio de Transporte, INVIAS, Archivo CORPES.

${ }^{5}$ Rausch, Jane. La Frontera de los Llanos en la historia de Colombia, 1830-1930 (Bogotá: Banco de la República, El Áncora Editores, 1999).

${ }^{6}$ Driver, Felix. "Imagining the tropics: views and visions of the tropical world", en Singapore Journal of Tropical Geography, vol. XXI, núm. 1, Singapore, Department of Geography, National University of Singapore and Blackwell Publishers Ltd, 2004, pp. 1-17.
} 
unos efectos sociales y materiales particulares ${ }^{7}$. Es posible evidenciar que en el caso de la integración de los Llanos, a través del río Meta, la implementación de dichos proyectos generó profundas transformaciones en el paisaje, aunque no logró el objetivo propuesto en su totalidad.

Los Llanos Orientales u Orinoquia colombiana estuvieron poblados por una gran diversidad de grupos indígenas en tiempos precolombinos. Estos grupos tenían extensas redes comerciales e interétnicas que comunicaban el Amazonas, los Andes, el Pacífico e incluso islas del Atlántico ${ }^{8}$; redes que los españoles aprovecharon para avanzar en la búsqueda nuevos territorios ${ }^{9}$. Antes de la Conquista, esta no era una región de frontera, sin embargo, muy temprano en el siglo XVI empieza a ser configurada como tal. Las descripciones iniciales de la región realizadas por misioneros jesuitas representaban "una dicotomía entre un presente negativo y un futuro positivo"10. Lo negativo relacionado con la baja fertilidad de sus suelos, el clima cálido y húmedo que describieron como malsano, sus poblaciones salvajes y su aislamiento con respecto al centro administrativo Colonial, del otro lado de la Cordillera de los Andes; y lo positivo se veía a través de la inmensidad de tierras planas que podrían ser productivas con arduo trabajo y pobladas por gente civilizada, y el río como una importante ruta de comunicación. Para González estas representaciones crearon un continuum discursivo desde el siglo XVI hasta el presente, a través del cual aún se ve y se piensa a los Llanos $^{11}$. Durán, lo llama herencias moderno-coloniales porque perpetúan la idea de un espacio inmenso y vacío, una frontera interna, que se ha configurado como uno de los discursos dominantes sobre la región ${ }^{12}$.

Las visiones del Meta han estado relacionadas principalmente con la navegabilidad del río, lo que ha permitido construir ideas sobre este como un eje para la integración en diferentes momentos de la historia. La integración, en su significado más literal, hace referencia a "hacer que algo o alguien pase a ser parte de un todo"13 y el Meta es el eje central con el que se piensa integrar una extensa y solitaria región con alto potencial productivo. De esta manera, durante La Colonia, las compañías religiosas en los Llanos vieron en el río una inmensa red de aguas que les permitirían llegar a distantes lugares para fundar hatos y haciendas donde los indígenas se iniciaran en

\footnotetext{
${ }^{7}$ Tsing, Anna. "Nature in the Making", en Crumley, Carole L, et al. (eds.), New Directions in anthropology and Environment (Walnut Creek, Lanham, New York, Oxford: Altamira Press, 2001), pp. 3-23.

${ }^{8}$ Gómez, Augusto. Indios, colonos y conflictos. Una historia regional de los Llanos Orientales. 1870-1970 (Bogotá: Pontificia Universidad Javeriana, Instituto Colombiano de antropología, 1991). Tovar Pinzón, Hermes, Op. cit., Rausch, Jane, Op. cit.

${ }^{9}$ Gómez, Augusto, Op. cit., Tovar Pinzón, Hermes, Op. cit.

${ }^{10}$ González Gómez, Lina Marcela. "El papel de las crónicas misionales coloniales en la configuración de los Llanos Orientales de Colombia y en la producción social de las diferencias", en Historia y Sociedad, vol. 29, Medellín, Universidad Nacional de Colombia, 2015, pp. 17-42.

${ }^{11}$ Ibid.

${ }^{12}$ Durán, Armando. "Herencias moderno-coloniales en actuales discursos sobre la Orinoquia colombiana", en EURE, vol. XXXVIII, núm.115, Santiago de Chile, Universidad Católica de Chile, 2012, pp. 195-217.

13 Diccionario de la Real Academia de la Lengua Española. Entrada integrar, http://dle.rae. $\mathrm{es} / \mathrm{w}=$ integrar\&o $=\mathrm{h}$.
} 
la vida cristiana y civilizada ${ }^{14}$; en el siglo XIX el río era una ruta comercial poco explorada y atractiva para comerciar con Europa, que incentivaría la colonización de la región y su productividad ${ }^{15}$; y en el siglo XX se reanuda su interés como ruta comercial para exportar alimentos, minerales, hidrocarburos y biocombustibles, expandiendo la frontera agraria a través de un sistema intermodal de transporte que conecte al Atlántico y al Pacífico ${ }^{16}$.

\section{Breve contexto regional}

El Meta es el río más caudaloso y extenso de la Orinoquia colombiana, tiene una longitud de $1.000 \mathrm{~km}$ desde su nacimiento en la Cordillera de Los Andes hasta su desembocadura en el río Orinoco. La cuenca del Meta, con un área de $105.000 \mathrm{~km}^{2}$, comprende las partes altas de la cordillera oriental (Sumapaz y Sierra Nevada del Cocuy) donde nacen la mayoría de sus afluentes; un área de piedemonte y una extensa área de sabanas inundables, sabanas bien drenadas y parte de la altillanura ${ }^{17}$; hace parte de la macrocuenca colombo-venezolana del Orinoco con un área de $991.587 \mathrm{~km}^{2}$, de los cuales $347.713 \mathrm{~km}^{2}$ le corresponden a Colombia, el $30.4 \%$ del territorio nacional ${ }^{18}$. El río corre de Occidente a Oriente el país, pasando por los departamentos del Meta, Casanare, Arauca y Vichada (Imagen 1).

El primer español en llegar a los Llanos fue Diego de Ordaz en 1532, quien buscando El Dorado “[...] remontó el Orinoco desde el mar [...] hasta los grandes raudales a la altura de Ayacucho, virando luego, guiado por el indígena Tanate, por las bocas del Meta hacia arriba"19. Federmán en 1538, proveniente de Coro (Venezuela), Hernán Pérez de Quesada en 1542 y su hermano Gonzalo Jiménez de Quesada llegaron desde el Altiplano Cundiboyacense (Colombia); todos recorrieron los Llanos en busca de El Dorado e hicieron fundaciones de pueblos a su paso que no perduraron mucho ${ }^{20}$. Antonio de Berrío, fue el primero en establecer una ruta entre Bogotá-Río CasanareMeta-Orinoco-Guyana-Trinidad entre 1584 y $1586^{21}$.

Con el avance de la colonización y fundación de pueblos, se fueron concediendo las encomiendas, que "[...] lo fueron más de nombre, pues generalmente no rindieron los frutos

\footnotetext{
${ }^{14}$ Silva Fajardo, Germán. Champanes, vapores y remolcadores. Historia de la navegación y la ingeniería fluvial Colombiana (Bogotá: Academia Colombiana de Historia de la Ingeniería y las Obras Públicas. Cuaderno de Historia, núm.1, 2009). Tovar Pinzón, Hermes. Relaciones y Visitas a Los Andes. S. XVI. Región de los Llanos (Bogotá: Universidad de Los Andes, 2010).

${ }^{15}$ Franco García, Roberto. Historia de Orocué (Bogotá: Kelt Colombia/Ecopetrol, 1997).

${ }^{16}$ Ospina Hernández, Mariano. La integración Fluvial de Suramérica (IFSA) (Bogotá: CIFSA, 1999).

${ }^{17}$ CORPES Orinoquia. Preplan de manejo de la cuenca hidrográfica del río Meta (Villavicencio: Corpes Orinoquia, 1998). Sin publicar.

${ }^{18}$ Romero Ruiz, Milton, et al. Ecosistemas de la Cuenca del Orinoco Colombiano (Bogotá: Instituto de Investigación de Recursos Biológicos Alexander von Humboldt, Instituto Geográfico Agustín Codazzi, 2004).

${ }^{19}$ Mejía Gutiérrez, Mario. Aventureros, cronistas y cientificos en la Orinoquia (Bogotá: Universidad Nacional de Colombia, 2004), p. 3.

${ }^{20}$ Franco García, Roberto, Op. cit.; Mejía Gutiérrez, Mario, Op. cit.

${ }^{21}$ Mejía Gutiérrez, Mario, Op. cit.
} 
El rio Meta y los proyectos para la integración de los Llanos Orientales colombianos...

(tributos) esperados por sus titulares, en virtud de las dificultades que estos encontraron para 'poner en policía' a sus encomendados" ${ }^{\text {"22 }}$. Por esta razón recurren a las misiones como estrategia de colonización.

Imagen 1. Área de estudio.

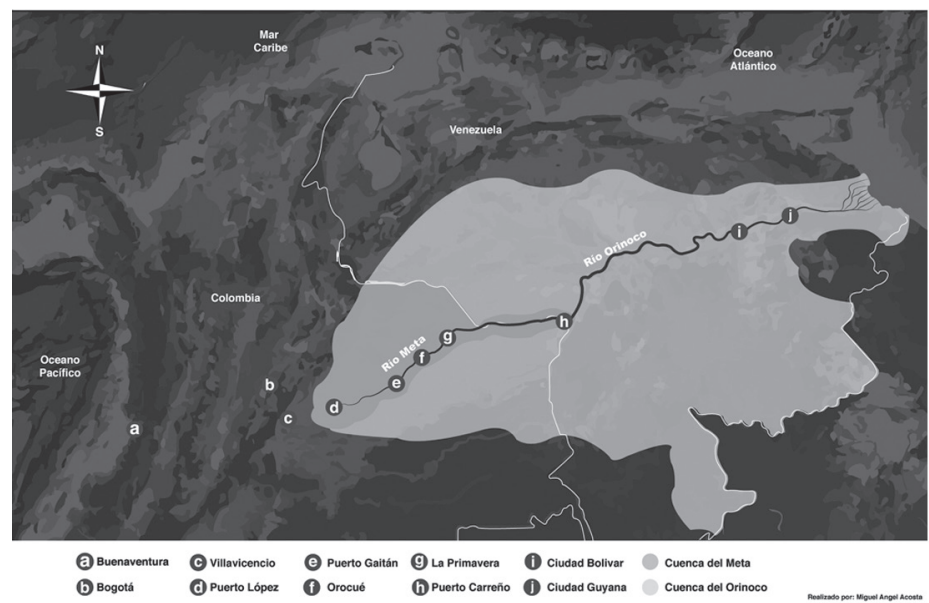

Fuente: Google Earth, modificado por Miguel Ángel Acosta S.

\section{Las misiones jesuitas}

Las misiones hicieron parte de las estrategias de colonización auspiciadas por La Corona, para aquellos lugares donde las formas más tradicionales (pueblos, encomiendas) no perduraban en el tiempo. En el caso de los Llanos, su población indígena descrita como caribe o salvaje y en su mayoría itinerante, su clima cálido y su difícil acceso desde Santa Fe, dificultaban el asentamiento de los españoles ${ }^{23}$. Ante esta situación, compañías religiosas tomaron la iniciativa para hacer presencia en aquellas regiones con la intención de hacerse cargo de la vida material y espiritual de los grupos indígenas Achaguas, Sálivas, Piapocos, Cuivas, Guahibos, entre otros; el proyecto consistía en fundar hatos y haciendas que les permitieran estar cerca de ellos e instruirlos en el cristianismo.

La compañía de mayor presencia en los Llanos fue la jesuita, su primera entrada fue en 1625 hasta que les retiraron sus facultades en 1628, y posteriormente ingresaron en 1659 hasta 1767, año de su expulsión ${ }^{24}$. Los jesuitas llegaron a los Llanos colombianos

\footnotetext{
${ }^{22}$ Gómez, Augusto, Op. cit.

${ }^{23}$ Rauch, Jane, Op. cit.

${ }^{24}$ Gómez, Augusto, Op. cit.; Cassani, Joseph. Historia de la provincia de la Compañía de Jesús en el Nuevo Reyno de Granada, t. I. (Imprenta, y librería de Manuel Fernández, frente de la Cruz de la Puerta Cerrada, 1741).
} 
desde los páramos de Chita y Pauto, bajando por los ríos Casanare, Cravo y Tocaría, y en el camino fundaron hatos y haciendas ${ }^{25}$. Encontraron numerosos y diversos grupos que vivían cerca de los ríos y en la inmensidad del Llano al que llamaban desierto. En este proceso de expansión y consolidación de las misiones, los ríos son parte central de las narrativas de los jesuitas dedicadas a describir las experiencias de la Compañía en los Llanos. Gumilla llama al Orinoco como Mar Dulce, por su inmensidad, innumerables bocas que desaguan en el mar, y la gran cantidad de afluentes que lo alimentan y le otorgan su tamaño e imponencia ${ }^{26}$. El padre Rivero resalta la abundancia de fuentes de agua: "La distancia que hay de ríos á ríos y de quebradas á quebradas es tan proporcionada, que á dos ó tres leguas de camino se suceden las unas á las otras"27.

La abundancia de ríos y quebradas, la mayoría navegables, pronto se convirtieron en una vía de comunicación entre tierras altas y bajas, y a lo largo de los Llanos en el recorrido por el Meta, el Guaviare y el Orinoco. Cassani menciona repetidas veces que la navegación de los ríos siempre era muy cómoda, solo en algunas ocasiones cuando el río crecía se hacía difícil o se corría riesgo cuando iban a ser atacados por grupos indígenas ${ }^{28}$. También Gumilla describe al Meta como un río tranquilo y amplio en el que no lograba percibirse ni la fuerza de sus aguas ni la profundidad de su cauce ${ }^{29}$. A partir de estos recorridos se van configurando los mapas de la región y los ríos marcan los puntos de habitación de las diferentes poblaciones ${ }^{30}$. Las cifras muestran la importancia de los ríos como hábitat principal de los indígenas pues en ellos se concentraba en 1556 , el $70 \%$ de las casas, mientras que en los valles estaba en $30 \%{ }^{31}$. Rivero expresa, para el caso de los hoy Llanos colombianos, que la idea fue siempre "reducir [a los indígenas] al Meta" o sus cercanías ${ }^{32}$. Llano adentro, como le dicen, era muy difícil lograr mantener el control.

En el avance del proyecto de fundación de hatos y haciendas, las misiones impulsaron profundos cambios en el paisaje. El paisaje como proceso y producto social, es reflejo inherente de las relaciones humanas y de estas con los no-humanos ${ }^{33}$, así, cuando las misiones introdujeron especies animales y vegetales europeas llevaron a nuevas formas de habitar el espacio (en pueblos y haciendas), de usar el suelo y de relacionarse con el entorno; en especial buscaban que las tradicionales y mayoritarias poblaciones itinerantes de los Llanos se establecieran en lugares permanentes. Las misiones logran poner las bases de lo que se empieza a conocer como el tradicional paisaje del hato

\footnotetext{
${ }^{25}$ Rivero, Juan de, Op. cit., Cassani, Joseph, Op. cit; Gumilla, José, Op. cit.; Ibíd.

${ }^{26}$ Gumilla, José, Op. cit.

${ }^{27}$ Rivero, Juan de, Op. cit.

${ }^{28}$ Cassani. Joseph, Op. cit., p. 150

${ }^{29}$ Gumilla, José, Op. cit., p. 55.

${ }^{30}$ Cassani. Joseph, Op. cit., p. 147.

${ }^{31}$ Tovar Pinzón, Hermes, Op. cit., p. 36.

${ }^{32}$ Rivero, Juan de, Op. cit., p. 33.

${ }^{33}$ Tsing, Anna, Op. cit.
} 
El rio Meta y los proyectos para la integración de los Llanos Orientales colombianos...

llanero $^{34}$, a la creación del llanero como habitante característico de la región, y las vacas y caballos como especies naturales de la región.

\section{Una nueva ruta para la exportación. Siglos XIX y XX}

Durante el siglo XIX, los gobiernos de Colombia hicieron esporádicos intentos de desarrollar la porción oriental de la República. Entre sus estrategias se contaron con la reanudación de las misiones, la promoción de la navegación a vapor por el río Meta, la construcción de caminos y el fomento de la inmigración, así como del crecimiento económico, mediante la introducción de nuevos cultivos, pastos mejorados y mejores razas vacunas ${ }^{35}$.

El río Meta a finales del siglo XIX e inicios del XX se convirtió en una fuente de sueños, promesas y proyectos que lograron momentáneamente integrar a la región de los Llanos a la Nueva República y al mundo. El río nuevamente fue pensado como un eje integrador de la región, esta vez con argumentos principalmente económicos que pretendían llevar prosperidad, construyendo y habilitando vías de comunicación que impulsaran actividades agrícolas y de obtención de recursos naturales (caucho, quina, plumas, sarrapia) con fines de exportación y actividades pecuarias para el mercado nacional.

Durante la segunda mitad del siglo XIX se da el auge agroexportador. Los diferentes gobiernos, principalmente de corriente liberal, estimularon la migración de personas a las zonas de frontera para explotar los bosques libremente y cultivar productos que tuvieran alta demanda en el exterior, delegando en los privados las dinámicas económicas del país ${ }^{36}$; se entregaron títulos de tierras a quienes trabajaron el campo y se construyó una infraestructura que facilitara el flujo de mercancías y capital ${ }^{37}$. Después de 1886, con el regreso de los conservadores al poder político, la explotación libre de los recursos se limitó en cantidad y extensión, retornaron las misiones, y se propició el proceso de acumulación de tierras; y aunque no se pierde el énfasis agroexportador, hay una mayor vigilancia y promoción desde el Estado para intervenir las actividades económicas ${ }^{38}$.

Los Llanos empiezan a hacer parte de una visión que pensaba este lugar como el futuro de Colombia ${ }^{39}$, un lugar de producción de alimentos para la Sabana de Bogotá y de productos para el mundo; el río Meta se convierte en una ruta fundamental para asegurar el crecimiento económico. Esta visión era alimentada por las informaciones

\footnotetext{
${ }^{34}$ Gómez, Augusto; Molina, Nathaly y Suárez, Carolina. "Vichada: éxodo y etnocidio indígena; el avance de la ganadería extensiva y de la colonización”, en Maguaré, vol. XXIX, núm. 1, Bogotá, Facultad de Ciencias Humanas Universidad Nacional de Colombia, 2012, pp. 75-121.

${ }^{35}$ Rausch, Jane. 1999, Op. cit., p. 15.

${ }^{36}$ Palacio, Germán. Fiebre de tierra caliente: una historia ambiental de Colombia 1850-1930 (Bogotá: Universidad Nacional de Colombia, Sede Amazonía: Ilsa: Saber y Gestión Ambiental, 2006).

${ }^{37}$ Ibid.

${ }^{38}$ Palacio, Germán, Op. cit.

${ }^{39}$ Rausch, Jane, Op. cit.
} 
de científicos que visitaron la región: Humboldt, quien recorrió el Orinoco y llegó a la boca del Meta en 1800, menciona que establecer una ruta de comunicación del Meta era importante para las regiones aledañas y el comercio de bienes como la sal ${ }^{40}$. Posteriormente en 1856, la Comisión Corográfica, Codazzi realiza la descripción del Casanare, y aunque es bastante pesimista al hablar de la región por su atraso, presencia de salvajes y mal clima, también habla de la potencialidad de la región para la producción agropecuaria, señalando que el desarrollo de vías de comunicación por tierra y río - específicamente el Meta- eran vitales para que se diera un cambio positivo ${ }^{41}$. Esta lectura entre la descripción fisiográfica y estética que hacen estos dos científicos, hacen parte de lo que Driver llama Vistas (Views) y visiones, en donde se proyectan las lecturas y las experiencias -incluso estéticas- que generan los paisajes sobre ellos, creando representaciones poderosas sobre un lugar ${ }^{42}$. Estas visiones alimentan los sueños y las promesas de gobernantes que plantearon políticas y proyectos de gobierno que hicieran posible la realización de las potencialidades de los Llanos. Los comerciantes también compartieron estas visiones y las concretaron, estableciendo rutas comerciales y mostrando las bondades que tendría para la región si fuera algo regular ${ }^{43}$.

La navegación comercial del río empieza a ser promovida por los gobiernos liberales después de la Independencia, sin embargo, el siglo XIX y los inicios del XX estuvieron marcados por un alto número de guerras civiles y problemas fronterizos con Venezuela, lo que impidió el avance y la consolidación de la navegación ${ }^{44}$; además de la influencia que ejercieron los comerciantes de Cartagena para impedir el despliegue de la navegación en el Meta por intereses económicos ${ }^{45}$. La navegación del Meta permitía traer productos desde Europa y Ciudad Bolívar (Venezuela) por el Orinoco hasta poblados como Orocué, Villavicencio y con gran esfuerzo hasta Bogotá y otras regiones de la Cordillera Oriental ${ }^{46}$.

El proyecto de navegación estaba vinculado a procesos de colonización de la región para integrar nuevos espacios productivos a la economía del país y del mundo. Esta iniciativa liderada por el gobierno iba dirigida a que nuevos colonos y empresarios se hicieran cargo de materializar el proyecto. Entonces, tres grupos externos tomaron las riendas de la integración, transformando nuevamente el paisaje. En el caso de la navegación, los empresarios introdujeron nuevas tecnologías como los buques

\footnotetext{
${ }^{40}$ Humboldt, Alexander Von. Del Orinoco al Amazonas: Viaje a las regiones equinocciales del Nuevo Continente (Barcelona: Editorial Labor, 1967).

${ }^{41}$ Codazzi, Agustín. Geografia física y política de la Confederación Granadina. Viaje de la Comisión Corográfica por el territorio de Casanare 1856, vol. III, t.1. Bogotá: Universidad Nacional de Colombia, U.P.T.C., Universidad del Cauca). Edición, análisis y comentarios por Augusto Gómez, Guido Barona Becerra y Camilo Domínguez.

${ }^{42}$ Driver, Felix, Op. cit.

${ }^{43}$ Franco Garcia, Roberto, Op. cit.

${ }^{44}$ Rausch, Jane, (1999), Op. cit.

${ }^{45}$ Franco García, Roberto, Op. cit.

${ }^{46}$ Ibid.
} 
a vapor que requerían de grandes cantidades de madera (cantidades a las que el gobierno les dio acceso en los bosques aledaños al río); el incremento de la carga implicó nueva infraestructura de puertos y bodegas de almacenamiento. Los colonos y los empresarios empezaron a cultivar tierras con productos comerciales como el café, a explotar el caucho, la sarrapia, las plumas de garza. El caso más claro para entender el proyecto de integración es el de José Bonett, empresario francés considerado como el primero en establecer una navegación regular por el Meta ${ }^{47}$. A Bonett el gobierno venezolano le otorgó una concesión especial para la libre navegación y transporte de mercancías en puertos francos en el Meta y Orinoco en 1880, y sus posibilidades de navegar el Meta estaban contempladas por las disposiciones de libre navegación que también tenía Colombia. Bonnet, además de navegante, fue un empresario con casas comerciales en Orocué y Villavicencio, y de cultivo de café. En 1884 obtuvo una nueva licencia para navegar el Meta, y en esta se especifican sus funciones: crear puertos y bodegas que facilitaran el transporte de las mercancías, le asignaron $30.000 \mathrm{Has}$ de terrenos baldíos sobre el eje del Meta para que produjera café, cacao y otros productos exportables, además de subsidios en dinero por cada viaje realizado. Todo iba muy bien hasta que estalló la Guerra de los Mil Días, cuando tomaron uno de los barcos de Bonnet y los destrozaron, lo que luego contribuyó a su quiebra ${ }^{48}$. La navegación por el Meta mantuvo alguna regularidad gracias a los incentivos que daba el gobierno, pero en 1930 se suspende casi definitivamente como resultado de la crisis económica de 1929 que desestimuló las exportaciones hacia Europa y Estados Unidos ${ }^{49}$.

Este proyecto con énfasis exportador también generó cambios en el paisaje. Adicional a la introducción de nuevas especies como el café, las economías extractivas de caucho, quina y sarrapia empiezan a dejar huella. Los nuevos ocupantes relegan más a los pobladores originales de la región restringiendo su movilidad -en el caso de las poblaciones itinerantes- o acceso a la tierra -por ejemplo, en las riberas del río-; sin olvidar la transformación de los ecosistemas que inician otro proceso de cambio bajo la lógica de las plantaciones y reforzamiento de la ganadería extensiva.

\section{Corredor Atlántico-Pacífico. Finales del siglo XX y XXI}

"El neoliberalismo, siempre en nombre del progreso y la libertad, encuentra su solución práctica, con la figura de la integración regional"50.

A finales del siglo XX el río vuelve a ser importante en los planes de desarrollo para la integración de la región. A lo largo del siglo XX se había intentado una integración regional a nivel de los países latinoamericanos, se firmaron varios acuerdos desde los 60 , pero muy pocos se llevaron a cabo y los realizados tuvieron poco impacto, parte central de los acuerdos era la construcción de rutas de comunicación vial (férrea,

\footnotetext{
${ }^{47}$ Rausch, Jane, Op. cit.

${ }^{48}$ Franco García, Roberto, Op. cit.

${ }^{49}$ Silva Fajardo, Germán, Op. cit.

${ }^{50}$ Achkar, Marcel yDomínguez, Ana. "La gestión del agua desde la geopolítica trasnacional y desde los territorios de la integración", en Soares, Denise; Vargas, Sergio y Nuño, María Rosa, La gestión de los recursos hidricos: realidades y perspectivas (Guadalajara: IMTA-Universidad de Guadalajara2008), p. 3.
} 
fluvial o carretera) $)^{51}$. A nivel suramericano entre 1987 y 1996 se realizaron diferentes exploraciones científicas que demostraban las posibilidades de integración fluvial de las cuencas del Orinoco, Amazonas y La Plata ${ }^{52}$; hasta el momento el único proyecto realizado ha sido el de La Plata con la construcción de la Hidrovía Paraguay-Paraná ${ }^{53}$. En el año 2000 se firma La Iniciativa para la Integración de la Infraestructura Regional Suramericana (IIRSA), aún vigente, la cual promueve la realización de proyectos de infraestructura que permitan conectar mejor a América del Sur entre ellos y con el resto del mundo, especialmente nuevas rutas hacia el Pacífico. El Meta hace parte del grupo cuatro con un proyecto para conectar el océano Atlántico con el Pacífico en un sistema de transporte intermodal vía río Orinoco-Meta-Bogotá-Buenaventura ${ }^{54}$.

El interés por el Meta no se reduce al río como vía navegable, sino que en el contexto mundial, la Orinoquia colombiana se convierte en un lugar atractivo para la ampliación de la frontera agrícola, en las visiones nacionales y globales ${ }^{55}$. Las visiones sobre esta región siguen reproduciendo las ideas de la Colonia como la de una extensa región con muy poca población y alto potencial productivo. Según Durán, un discurso hegemónico que defiende el gobierno y los gremios empresariales de palmeros, ganaderos, arroceros y petroleros ${ }^{56}$. De esta manera, “[...] la Orinoquia hace parte de las regiones de Colombia que están en fase de formación: un lienzo en blanco para ser pintado, un territorio abierto a la exploración y a la colonización, lleno de oportunidades para la iniciativa privada en donde, a diferencia de la Amazonia, no existen mayores restricciones ambientales"57. Así lo expresa claramente el expresidente Uribe:

Pero miren: le veo a esa Orinoquía [sic] un futuro ya inmediato en el agro. En esos $600.000 \mathrm{~km}$ diríamos que hay 350.000 de selva y 250.000 de sabanas. ¿Ustedes saben lo importante que es para el mundo agropecuario tener allí $250.000 \mathrm{~km}$ planos, sin piedra, listicos para cultivar y sin el obstáculo ecológico de que hay que llegar con el hacha? ${ }^{58}$.

El proyecto actual de navegabilidad y de desarrollo se olvida de los Llanos Orientales como lugar del hato llanero; ahora es la Orinoquia y dentro de ella la Altillanura (ubicada especialmente en los departamentos del Meta y Vichada). Con la intención

\footnotetext{
51 Ospina Hernández, Mariano, (1999a), Op. cit.

${ }^{52}$ Georgescu-Pipera, Constantino y Georgescu-Pipera, Paul. Los ríos de la integración suramericana (Caracas: Universidad Simón Bolívar, Instituto de Altos Estudios de América Latina, 1984).

${ }^{53}$ Ospina Hernández, Mariano, (1999b), Op. cit.

54 UNASUR, Cosiplan. Foro técnico IRSSA, Eje Andino, http://www.iirsa.org/Page/ PageDetail?id=116\&menuItemId=68 (9 de noviembre del 2015).

55 Jiménez, M. "Valorización capitalista de la Orinoquia colombiana. División territorial del trabajo e inserción en la acumulación transnacional”, en Ciencia Política; núm. 13, 2012, pp. 150-180.

${ }^{56}$ Durán, Armando, Op. cit.

${ }^{57}$ Rodríguez, Manuel, et al. La mejor Orinoquia que podemos construir: elementos para la sostenibilidad ambiental del desarrollo (Bogotá: Corporinoquia, Universidad de los Andes, Foro Nacional Ambiental y Fescol, 2009).

${ }^{58}$ Uribe citado en Durán, Armando, Op. cit., p. 202
} 
de hacer productiva a la región el gobierno central creó en 1969 el Centro de Investigación Carimagua para generar conocimiento aplicado al mejoramiento de la ganadería y la producción agrícola ${ }^{59}$; asimismo en 1971 se crea el Centro Las Gaviotas, con la finalidad de demostrar el potencial de desarrollo de actividades productivas con plantaciones de bosques multipropósito ${ }^{60}$. Estos centros de investigación demostraron que la ciencia y la tecnología pueden transformar esta zona improductiva en una muy productiva. También el descubrimiento de petróleo y el inicio de plantaciones de palma de aceite ${ }^{61}$ contribuyeron a consolidar esta visión de región surgida en el siglo XIX como futuro de Colombia. En los ochenta, bajo el gobierno de Betancur, se crea el programa Marandúa, una ciudad en el Vichada soñada como una Nueva Colombia basada en los antecedentes de Carimagua y Gaviotas, que estimularía la colonización de nuevas tierras en un proceso acompañado por las diferentes instituciones del Estado $^{62}$; el interés del proyecto buscaba ampliar la frontera agrícola con la producción campesina y ejercer soberanía en las regiones de frontera.

En 1985 el Ministerio de Obras Públicas le solicitó a Francisco Pacheco Ceballos, ingeniero hidráulico, la realización de un estudio técnico para la rehabilitación de la navegación en el río Meta ${ }^{63}$. Pacheco menciona que "[...] el transporte fluvial entre Puerto López a Ciudad Bolívar, Matanza o Puerto Ordaz constituye la base de la integración y en definitiva la mejor justificación para el desarrollo general de la cuenca" ${ }^{64}$, teniendo en cuenta que la falta de vías de comunicación es el principal obstáculo para el desarrollo socioeconómico de la región y que “[...] la navegabilidad del río permitirá bajar los costos del transporte fluvial, incentivar la ganadería, mejorar las técnicas de la ganadería, reducir los monopolios de comercialización, mejorar los planes de inversión" ${ }^{65}$. Sin embargo, al igual que la filosofía de Marandúa, hace énfasis en que el proyecto será exitoso en la medida en que las diferentes entidades del Estado hagan una mayor presencia, ofreciéndoles a los pobladores y nuevos colonos "crédito, seguridad social, soberanía y evitar que sea manejada la región por los grupos subversivos" 66 .

En los años 90 hay un cambio importante en términos de la finalidad de la integración con respecto a los de la década anterior. Los proyectos de rehabilitación de la

\footnotetext{
${ }^{59}$ García Durán, Obed. "Carimagua: La investigación y el desarrollo en ecosistemas de baja fertilidad”, en Revista Colombiana de Ciencias Pecuarias, núm. 22, 2009, pp. 74-78.

${ }^{60}$ Lugari Castrillón, Paolo. "Centro Las Gaviotas. El futuro en las manos”, en Torres Acevedo, Carlos Arturo (ed.), Los Llanos de Colombia (Bogotá: Litografía Arco, 1986), pp.187-189.

${ }^{61}$ Rausch, Jane. "La mirada desde la periferia: desarrollos en la historia de la frontera colombiana, desde 1970 hasta el presente", en Fronteras de la Historia, núm. 8, 2003, pp. 251-60.

${ }^{62}$ Londoño, Bernardo. "Programa Marandúa", en Torres Acevedo, Carlos Arturo (ed.), Los Llanos de Colombia (Bogotá: Litografía Arco, 1986), pp. 191-192.

${ }^{63}$ Ministerio de Transporte (MT). Estante 16-3. Nivel 2. Folios 1-23. Bogotá, 1985.

${ }^{64}$ Ministerio de Transporte (MT). Estante 34-3. Nivel 2. Folios 1-144. Bogotá, 1987. Folio 33.

${ }^{65}$ Ministerio de Transporte (MT). Estante 34-3. Nivel 2. Folios 1-144. Bogotá, 1987. Folio 35.

${ }^{66}$ Ministerio de Transporte (MT). Estante 34-3. Nivel 2. Folios 1-144. Bogotá, 1987. Folio 139.
} 
navegabilidad del río posteriores a 1990 responden a los intereses de promover economías de escala basadas en la producción agroindustrial, la explotación de minerales y petróleo ${ }^{67}$. En este periodo Colombia entra al neoliberalismo y se incentiva la apertura de los mercados y un profundo cambio en las funciones del Estado. Argumentando el fracaso de la política de sustitución de importaciones que llevaron a la creación de una economía cerrada ${ }^{68}$, el país se apresura para integrarse al mercado global sin tener la preparación, producción ni calidad requeridas en los mercados externos, lo cual terminó por crear una crisis económica en la producción agrícola e industrial nacional ${ }^{69}$. Achkar y Domínguez dicen que en el neoliberalismo "[...] la integración es el nuevo paradigma de solución, ya que los Estados-Territorios constituyen el freno a la libre circulación del flujo de capitales y retardan la aceleración del consumo. Ante ello, la solución implica la disolución de barreras"70.

En esta época el desarrollo de proyectos de infraestructura es determinante para la región. El encargado de coordinar es el Consejo Nacional de Política Económica y Social (CONPES) y donde la Altillanura y el río Meta son protagonistas ${ }^{71}$. Y es el momento en que se empiezan a realizar el mayor número de estudios y obras en el río. En 1997 se realiza el estudio para la construcción del muelle intermodal La Banqueta (Puerto López, Meta) ${ }^{72}$ que permitió conectar el modo fluvial y el carretero; y en 1999 se contrató un estudio de demanda financiado por el BID y el PNUD que tenía como finalidad rehabilitar el río y entregarlo en concesión ${ }^{73}$. En el año 2000, se firma la iniciativa IIRSA donde uno de los proyectos es crear un corredor vial intermodal que comunique el Atlántico con el Pacífico ${ }^{74}$. Esta iniciativa fue seguida por las primeras obras de encauzamiento en el año $2006^{75}$. En 2013 se realiza una actualización de los diseños y los estudios técnicos realizado por la Universidad del Norte $^{76}$; y se publican el Plan Maestro de Transporte para la Altillanura y el Plan

\footnotetext{
${ }^{67}$ Ministerio de Agricultura y Desarrollo Rural. El Renacimiento de la Alta Orinoquia de Colombia. Un megaproyecto para el mundo (Bogotá: Ministerio de Agricultura y Desarrollo Rural, 2004).

${ }^{68}$ Departamento Nacional de Planeación. La revolución pacifica. Plan de desarrollo económico y social, 1990-1994 (Santa Fe de Bogotá: Departamento Nacional de Planeación, 1991).

${ }^{69}$ González, Jorge Iván. "Entre la sustitución de importaciones y la apertura", en Desarrollo económico $y$ social en Colombia. Siglo XX (Bogotá: Universidad Nacional de Colombia, Facultad de Ciencias Económicas, 2001).

${ }^{70}$ Achkar, Marcel y Domínguez, Ana, Op. cit., p. 4.

${ }^{71}$ CONPES 2691 de 1994, CONPES 3396 del 2005, CONPES 3510 del 2008, CONPES 3797 del 2014.

${ }^{72}$ Ministerio de Transporte (MT). Estante 32. Carpeta 6711. Caja 362. Folios 1-146. Bogotá, 1997.

${ }^{73}$ Cal y Mayor y Asociados. Estudio de demanda del río meta. Propuesta técnica presentada por Cal y Mayor y Asociados, Econometría para el Programa de las Naciones Unidas para el Desarrollo (Santafé de Bogotá: C\&M, Econometría, 2000). Sin publicar. Disponible en Colección CORPES de la Biblioteca Luis Ángel Arango, Bogotá.

74 UNASUR, Cosiplan. Foro técnico IRSSA, cumbre presidentes, http://www.iirsa.org/Page/ Detail?menuItemId=41 (17 de noviembre del 2015).

${ }^{75}$ Autoridad Nacional de Licencias Ambientales (ANLA), Expediente 2693 de 2006.

${ }^{76}$ Instituto Nacional de Vías (INVIAS). Archivo proporcionado por la sección de Estudios Técnicos, 2013.
} 
El rio Meta y los proyectos para la integración de los Llanos Orientales colombianos...

Maestro de Navegación Fluvial ${ }^{77}$. A pesar de todos estos estudios, el proyecto se ha visto truncado por problemas de presupuesto y la inestabilidad política del gobierno venezolano.

La finalidad de la integración es descrita claramente por el estudio de la Universidad del Norte:

\begin{abstract}
Desde hace unos años, con los hallazgos y explotaciones de petróleo en los departamentos de Casanare, Meta y Vichada y la reciente identificación de utilidad del suelo para la siembra industrial de palma africana, soya, maíz, caña de azúcar y productos forestales en los departamentos de Meta y Vichada, se hace necesario, integrar la cuenca del río Meta, a la región más desarrollada del país, y además, tener la posibilidad de comunicarse a través del río Orinoco, para tener intercambios comerciales con Venezuela. Además con esto, se daría la mejora directa de las condiciones de vida de la población, las cuales son fundamentales para que la región pueda avanzar en el camino del desarrollo económico, su sostenibilidad ambiental y social ${ }^{78}$.
\end{abstract}

Es claro que la finalidad de la integración se centra en la importancia de los recursos naturales disponibles. Sassen menciona que este es un proceso que empieza a gestarse desde los ochenta cuando el establecimiento del mercado financiero llega a la cima y se posesiona con la autoridad máxima para manejar los mercados, y una de sus consecuencias es que los recursos naturales se vuelven más importantes que la gente ${ }^{79}$. Achkar y Domínguez plantean en este contexto una estrategia que consiste en integrarse al mundo es decir integrarse al mercado, de tal manera que las mejoras directas que van a beneficiar a la población local de la Orinoquia, estarían únicamente mediadas por las relaciones comerciales y serían beneficiosas en cuanto hubiera un mercado establecido.

La lectura de los proyectos de integración y navegabilidad del río permite ver nuevamente un gobierno central que piensa y toma las grandes decisiones de la región, con estrategias que buscan la colonización y la inversión de grandes capitales. De esta manera, los campesinos (llaneros y colonos), indígenas y habitantes urbanos de la región van desapareciendo en medio de un nuevo paisaje agroindustrial y de explotación petrolera que se está consolidando y que es nuevamente dejado en manos privadas como en el siglo XIX.

\title{
Conclusión
}

Los ríos han sido importantes para todas las sociedades del mundo como ejes articuladores para el poblamiento, el transporte, como fuentes de alimentos y consideraciones culturales como espacios sagrados, entre otros. Aunque este artículo se centra en las visiones externas sobre el río, lo que se va haciendo evidente es que

\footnotetext{
${ }^{77}$ Ministerio de Transporte (MT) Archivos proporcionados por la Subdirección Marítima y Fluvial.

${ }^{78}$ Instituto Nacional de Vías (INVIAS), Op. cit., p. 121. Cursiva de la investigación.

${ }^{79}$ Sassen, Saskia. Expulsions (USA: Harvard University Press, 2014).
} 
el río no es simplemente un escenario sobre el cual discurren los acontecimientos. En el caso del río Meta, el ser concebido como centro de las visiones y proyectos de integración de la región, constituye una parte importante para entender el proceso histórico de los Llanos, donde el río hace parte de los agentes que intervinieron en la forma como sucedieron los diferentes acontecimientos: sus características físicas que facilitan la navegación, su dinámica de aguas altas y bajas, el ser un referente para el poblamiento, también como fuente de alimentos para las personas e insumos (madera principalmente) para la navegación. La forma en la que el río fue observado y pensado, la relación que la sociedad colonial, republicana y contemporánea estableció con el río, incidió en las decisiones que afectaron a la región. Aunque en este texto no se planteó como una historia ambiental del Meta, sí sería importante profundizar en el análisis de las relaciones que las sociedades establecieron con el río para entender de manera más integral su papel en la región a través del tiempo.

Como se muestra en el texto una constante importante de la región es el planteamiento de la intervención externa como solución a los problemas de aislamiento y condición de frontera de la región, lo que ha terminado por fijar la idea de que esta necesita ser asistida para cambiar su situación. Bajo esta perspectiva, es notorio que los proyectos planteados y las soluciones propuestas se realizan a partir de ideas traídas de otros lugares, experiencias de otras regiones que no necesariamente se ajustan a las condiciones de la región a ser intervenida. Pero más importante que esto, es que dichos proyectos partieron de la negación de lo propio, de la diversidad intrínseca de los Llanos: sus ecosistemas, sus poblaciones locales, y su proceso histórico particular. Pensar en una región vacía, tanto de gente como de actividades productivas, crea un escenario para la intervención sin conflictos, sin problemas y sin posibilidades de oposición o alternativas.

Ninguno de los proyectos aquí analizados logró plenamente su objetivo, y no por esto deberían entenderse como fracaso, sin embargo, es importante reconocer que han tenido impactos a diferentes niveles en la región, transformando profundamente su paisaje - de sabanas y bosques de galería a hatos ganaderos y posteriormente a agroindustria y petróleo-. Ferguson lo llama side effects, efectos de proyectos que fallaron pero lograron establecer una nueva racionalidad en un lugar determinado que lo hacía depender de un centro administrativo mayor. El cambio del paisaje es entonces la expresión de un cambio profundo en las relaciones de las diferentes sociedades con su entorno, que ha sido dirigido por las intervenciones externas que han introducido nuevas actividades, nuevas poblaciones, nuevas ideas a las que las poblaciones de la región (tradicionales y colonas) se han ido adaptando de diversas formas, y donde no se sabe claramente cuál ha sido el impacto ambiental y social de dichas transformaciones que se relacionan con el deterioro del río, la deforestación de bosques de galería, las inundaciones que afectan cultivos, los efectos de una ganadería naturalizada como si hubiera estado siempre presente, el consumo de agua de los cultivos agroindustriales, entre otros. Como se mencionó, los resultados no fueron los esperados, pero al mismo tiempo es claro que quienes lideraron las intervenciones externas tampoco asumen la responsabilidad por las afectaciones negativas, haciendo de dichos resultados la excusa para nuevas intervenciones. 
El rio Meta y los proyectos para la integración de los Llanos Orientales colombianos...

\section{Bibliografía}

\section{Fuentes primarias}

\section{Archivos}

Archivo General de la Nación (AGN). Mapas-planos: sr.8, V. 22, R.53. Mapa de la Provincia y Misiones de la Compañía de Jesús del Nuevo Reyno de Granada

Autoridad Nacional de Licencias Ambientales (ANLA). Expediente 2693 de 2006.

CORPES Orinoquia. Preplan de manejo de la cuenca hidrográfica del río Meta. Villavicencio: Corpes Orinoquia, 1998. Sin publicar. Disponible en la colección Corpes de la Luis ángel Arango, Bogotá.

Instituto Nacional de Vías (INVIAS). Archivo proporcionado por la sección de Estudios Técnicos. 2013.

Ministerio de Transporte (MT). Estante 16-3. Nivel 2. Folios 1-23. Bogotá, 1985

(MT). Estante 34-3. Nivel 2. Folios 1-144. Bogotá, 1987.

(MT). Estante 32. Carpeta 6711. Caja 362. Folios 1-146. Bogotá, 1997.

(MT). Archivos proporcionados por la Subdirección Marítima y Fluvial.

\section{Libros}

Cassani, Joseph. Historia de la provincia de la Compañía de Jesús en el Nuevo Reyno de Granada, t. I. Colombia: Imprenta, y librería de Manuel Fernández, frente de la Cruz de la Puerta Cerrada, 1741.

Departamento Nacional de Planeación. La revolución pacífica. Plan de desarrollo económico y social, 1990-1994. Santa Fe de Bogotá: Departamento Nacional de Planeación, 1991.

Gumilla, José. El Orinoco ilustrado: historia natural civil y geográfica de este gran río y de sus caudalosas vertientes. Bogotá: Ministerio de Educación Nacional de Colombia, 1944.

Humboldt, Alexander Von. Del Orinoco al Amazonas: Viaje a las regiones equinocciales del nuevo continente. Barcelona: Editorial Labor, 1967. 


\section{Fuentes secundarias}

\section{Libros}

Cal y Mayor y Asociados. Estudio de demanda del río meta. Propuesta técnica presentada por Cal y Mayor y Asociados, Econometría para el Programa de las Naciones Unidas para el Desarrollo. Santafé de Bogotá: C\&M, Econometría, 2000. (Sin publicar). Disponible en Colección CORPES de la Biblioteca Luis Ángel Arango, Bogotá.

Codazzi, Agustín. Geografía física y politica de la Confederación Granadina. Viaje de la Comisión Corográfica por el territorio de Casanare 1856. Bogotá: Universidad Nacional de Colombia, U.P.T.C., Universidad del Cauca, vol. III, t. I. Edición, análisis y comentarios por Augusto Gómez, Guido Barona Becerra y Camilo Domínguez

Franco García, Roberto. Historia de Orocué. Bogotá: Kelt Colombia/Ecopetrol, 1997.

Georgescu-Pipera, Constantino y Georgescu-Pipera, Paul. Los ríos de la integración suramericana. Caracas: Universidad Simón Bolívar, Instituto de Altos Estudios de América Latina, 1984.

Gómez, Augusto. Indios, colonos y conflictos. Una historia regional de los Llanos Orientales. 1870-1970. Bogotá: Pontificia Universidad Javeriana, Instituto Colombiano de antropología, 1991.

Mejía Gutiérrez, Mario. Aventureros, cronistas y científicos en la Orinoquia. Bogotá: Universidad Nacional de Colombia, 2004

Ministerio de Agricultura y Desarrollo Rural. El Renacimiento de la Alta Orinoquia de Colombia. Un megaproyecto para el mundo. Bogotá: Ministerio de Agricultura y Desarrollo Rural, 2004.

Ospina Hernández, Mariano. La integración Fluvial de Suramérica (IFSA), t. I y II. Bogotá: CIFSA, 1999a.

Palacio, Germán. Fiebre de tierra caliente: una historia ambiental de Colombia 1850-1930. Bogotá: Universidad Nacional de Colombia, Sede Amazonía: Ilsa: Saber y Gestión Ambiental, 2006.

Pérez, Hipólito. Los jesuitas en Casanare. Bogotá: Prensas del Ministerio de Educación Nacional, 1952. 
El rio Meta y los proyectos para la integración de los Llanos Orientales colombianos...

Rausch, Jane. A Tropical Plains Frontier: the Llanos of Colombia, 1531-1831. Albuquerque: University of New Mexico Press, 1984.

Rausch, Jane. La frontera de los Llanos en la historia de Colombia. (1830-1930). Santafé de Bogotá: Banco de la República, El Ancora Editores, 1999.

Rivero, Juan de. Historia de las Misiones: de los Llanos de Casanare y los Ríos Orinoco y Meta. Bogotá: Presidencia de la República, 1956.

Rodríguez, Manuel, et al. La mejor Orinoquia que podemos construir: elementos para la sostenibilidad ambiental del desarrollo. Bogotá: Corporinoquia, Universidad de los Andes, Foro Nacional Ambiental y Fescol, 2009.

Sassen, Saskia. Expulsions. USA: Harvard University Press, 2014.

Silva Fajardo, Germán. Champanes, vapores y remolcadores. Historia de la navegación y la ingeniería fluvial Colombiana. Bogotá: Academia Colombiana de Historia de la Ingeniería y las Obras Públicas. Cuaderno de Historia núm. 1, 2009.

Tovar Pinzón, Hermes. Relaciones y Visitas a Los Andes. Siglo XVI. Región de los Llanos. Bogotá: Universidad de Los Andes, 2010.

\section{Capítulos de libros}

Achkar, Marcel y Domínguez, Ana. "La gestión del agua desde la geopolítica trasnacional y desde los territorios de la integración", en Soares, Denise; Vargas, Sergio y Nuño, María Rosa, La gestión de los recursos hídricos: realidades y perspectivas. Guadalajara: IMTA-Universidad de Guadalajara, 2008.

González, Jorge Iván. "Entre la sustitución de importaciones y la apertura”, en Desarrollo económico y social en Colombia. Siglo XX. Bogotá: Universidad Nacional de Colombia, Facultad de Ciencias Económicas, 2001.

Londoño, Bernardo. "Programa Marandúa”, en Torres Acevedo, Carlos Arturo (ed.), Los Llanos de Colombia. Bogotá: Litografía Arco, 1986.

Lugari Castrillón, Paolo. "Centro Las Gaviotas. El futuro en las manos", en Torres Acevedo, Carlos Arturo (ed.), Los Llanos de Colombia. Bogotá: Litografía Arco, 1986.

Tsing, Anna. "Nature in the Making", en Crumley, Carole L. et al. (eds.) New Directions in anthropology and Environment. Walnut Creek, Lanham, New York, Oxford: Altamira Press, 2001. 


\section{Artículos en revistas}

Castañeda Vanegas, Orlando. "Proceso de investigación, innovación y desarrollo sistema transporte fluvial río Meta”, en Anales de Ingeniería, vol. CXXVI, núm. 928, 2013.

Driver, Felix. "Imagining the tropics: views and visions of The tropical world", en Singapore Journal of Tropical Geography, vol. XXI, núm. 1, Singapore, Department of Geography, National University of Singapore and Blackwell Publishers Ltd, 2004.

Durán, Armando. "Herencias moderno-coloniales en actuales discursos sobre la Orinoquia colombiana", en EURE, vol. XXXVIII, núm. 115, Santiago de Chile, Universidad Católica de Chile, 2012.

García Durán, Obed. “Carimagua: La investigación y el desarrollo en ecosistemas de baja fertilidad”, en Revista Colombiana de Ciencias Pecuarias, núm. 22, 2009.

Gómez, Augusto; Molina, Nathaly y Suárez, Carolina. "Vichada: éxodo y etnocidio indígena; el avance de la ganadería extensiva y de la colonización”, en Maguaré, vol. XXIX, núm. 1, Bogotá, Facultad de Ciencias Humanas Universidad Nacional de Colombia, 2012.

González Gómez, Lina Marcela. "El papel de las crónicas misionales coloniales en la configuración de los Llanos Orientales de Colombia y en la producción social de las diferencias", en Historia y Sociedad, núm. 29, Medellín, Universidad Nacional de Colombia, 2015.

Ospina Hernández, Mariano. "La Integración Fluvial de Sur América: ingeniería civil y desarrollo sostenible”, en Ingeniería Civil, núm. 116, Madrid, 1999 b.

Rausch, Jane. "La mirada desde la periferia: desarrollos en la historia de la frontera colombiana, desde 1970 hasta el presente”, en Fronteras de la Historia, núm. 8, 2003.

\section{Publicaciones en Internet}

UNASUR, Cosiplan. Foro técnico IRSSA, cumbre presidentes, http://www.iirsa.org/ Page/Detail?menuItemId $=41$.

UNASUR, Cosiplan. Foro técnico IRSSA, Eje Andino, http://www.iirsa.org/Page/Pag eDetail?id=116\&menuItemId $=68$. 